Artículo científico

Volumen 31(2):445-459. Mayo-agosto, 2020

e-ISSN 2215-3608, doi:10.15517/am.v31i2.39046

http://www.revistas.ucr.ac.cr/index.php/agromeso

\title{
Monitoreo de Spodoptera spp. en caña de azúcar: uso de trampas con feromonas sexuales ${ }^{1}$
}

\section{Spodoptera spp. monitoring in sugarcane: use of sex pheromone traps}

\author{
José Daniel Salazar-Blanco ${ }^{2}$ Eduardo Cadet-Piedra², Francisco González-Fuentes ${ }^{3}$
}

1 Recepción: 18 de setiembre, 2019. Aceptación: 17 de diciembre, 2019. Este trabajo formó parte del Proyecto de investigación realizado en Cañas, Guanacaste de manera conjunta entre la Liga Agrícola Industrial de la Caña de Azúcar (LAICA) y ChemTica Internacional, S.A., Costa Rica.

2 Liga Agrícola Industrial de la Caña de Azúcar (LAICA), Departamento de Investigación y Extensión de la Caña de Azúcar (DIECA). 23301000, San José, Costa Rica. jsalazar@laica.co.cr (http://orcid.org/0000-0001-9656-684X); ecadet@laica.co.cr (https://orcid.org/0000-00015043-485X).

${ }^{3}$ ChemTica Internacional S.A., 640-3100, Heredia, Costa Rica. francisco_gonzalez@chemtica.com (http://orcid.org/0000-0001-8561-0411).

\section{Resumen}

Introducción. El gusano cogollero, Spodoptera frugiperda, es una de las plagas más devastadoras a nivel mundial. Aunque de origen americano, este insecto amenaza la seguridad alimentaria de todo el orbe. La razón es que su polifagia y adaptabilidad se combinan con los efectos del cambio climático. En Costa Rica las especies del orden Spodoptera son consideradas plagas menores en el cultivo de la caña de azúcar; no obstante, existe un desconocimiento sobre la dinámica poblacional de polillas de este género. Objetivo. Monitorear las poblaciones de las especies $S$. frugiperda, S. sunia y S. exigua en una plantación de caña de azúcar. Materiales y métodos. El estudio se efectuó en una plantación de caña de azúcar variedad CC 01-1940, ubicada en Guanacaste, Costa Rica, entre febrero de 2018 y mayo de 2019. Se emplearon trampas con feromonas sexuales formuladas para S. frugiperda, S. sunia y S. exigua, cada feromona correspondió a un tratamiento y se colocaron diez repeticiones de cada uno. Se comparó la abundacia de cada especie y se correlacionó con distintas variables climatológicas. Resultados. El mayor porcentaje de capturas correspondió a $S$. frugiperda, seguido por $S$. sunia, mientras que las capturas de $S$. exigua fueron marginales. Tanto para $S$. frugiperda como para $S$. sunia, los meses de la estación seca favorecieron mayores poblaciones, por lo que factores como mayor temperatura promedio, amplitud térmica y menor humedad relativa se asociaron con mayores capturas en las trampas. Conclusiones. Aumentos en la temperatura promedio y disminución de periodos de lluvia, podrían incrementar la incidencia del género Spodoptera como plagas en la producción cañera.

Palabras claves: Saccharum spp., cambio climático, feromonas sexuales.

\begin{abstract}
Introduction. The fall armyworm, Spodoptera frugiperda, is one of the world's most devastating pests. Although originally from the Americas, this insect has become a global food security threat. The reason is that its polyphagia and adaptability combine with the effects of climate change. In Costa Rica, the species of the Spodoptera order, are
\end{abstract}


considered minor pests in sugarcane production. Nevertheless, there is a knowledge gap in population dynamics of the moths of this genus. Objective. Monitor the populations of S. frugiperda, S. sunia, and S. exigua species in a sugarcane field. Materials and methods. The study was conducted in a sugarcane plantation of the variety CC 01-1940, located in Guanacaste, Costa Rica between February 2018 and May 2019. Sexual pheromone-baited traps formulated for S. frugiperda, S. sunia, and S. exigua were used, each pheromone corresponded to a treatment and ten replicates of each one were established. The abundance of each species was compared and correlated with several climatological variables. Results. The highest percentage of catches corresponded to $S$. frugiperda, followed by $S$. sunia, while the captures of $S$. exigua were marginal. For both $S$. frugiperda and $S$. sunia, the dry season months favored larger populations, so factors such as higher average temperature, thermal amplitude, and lower relative humidity were associated with higher captures in the traps. Conclusions. Increases in the average temperature and decrease in rainfall periods could increase the incidence of Spodoptera genus as pests in sugarcane production.

Keywords: Saccharum spp., climate change, sex pheromones.

\section{Introducción}

El gusano cogollero del maíz, Spodoptera frugiperda J.E. Smith, es un lepidóptero polífago originario de las Américas. Aunque se encuentra ampliamente distribuido en Norte y Sur América, en los últimos años se ha convertido en una amenaza para la seguridad agroalimentaria mundial, con su presencia en países africanos y asiáticos (Baudron et al., 2019). Debido a la capacidad de alimentarse de un gran número de especies de plantas, su alta proliferación y su habilidad de migrar grandes distancias, este insecto plaga ha sido capaz de causar pérdidas cuantiosas en gran cantidad de cultivos, particularmente gramíneas hacia las cuales presenta preferencia (Sparks, 1979). Entre los cultivos que afecta el gusano cogollero se encuentran el maíz, el arroz, el sorgo y la caña de azúcar. En el caso de la caña de azúcar es considerado una plaga menor, pero puede llevar a la pérdida de rendimiento, debido a la defoliación y que en el caso de plantas recién germinadas puede llevar a la muerte por el corte del tallo (Salazar et al., 2017).

La aparición de infestaciones de $S$. frugiperda en caña de azúcar en países como la India, ha llamado particularmente la atención, pues si bien los niveles de infestación son bajos comparados con los del maíz dulce, la capacidad de desarrollo de esta plaga y la relación filogenética entre ambos cultivos han generado preocupación entre los productores (Srikanth et al., 2018; Chormule et al., 2019). A nivel de Costa Rica, S. frugiperda, es una plaga que se presenta en todas las áreas productoras de caña de azúcar del territorio nacional, especialmente en campos rotados con arroz anegado, en los que en la mayoría de los casos logra ser manejada sin dificultad, a través de métodos de control biológicos, químicos y culturales principalmente (LAICA, 2019). No obstante, existe un vacío en el conocimiento respecto a sus hábitos de vuelo, así como la presencia o ausencia de otras especies relacionadas. Por ejemplo, se ha observado que especies como $S$. sunia Guenee y S. exigua Hubner, asociadas a cucurbitáceas como el melón y sandía, remolacha y algodón, respectivamente, son también observadas en plantaciones de caña de azúcar (LAICA, 2019).

Ante el efecto dañino que representa el gusano cogollero en plantaciones agrícolas a nivel mundial, la FAO ha determinado que la mejor herramienta de monitoreo para determinar las poblaciones y migraciones de Spodoptera, es el uso de trampas con señuelos de feromonas (FAO y CABI, 2019). Las hembras de $S$. frugiperda, así como muchas especies del género Spodoptera, utilizan compuestos volátiles como señales químicas sexuales para la atracción de machos, por lo que estos compuestos son sintetizados químicamente, aplicados en dispensadores y colocados en el campo para la atracción y captura específica de los machos de la especie a la cual corresponde la feromona. 
En el caso de caña de azúcar, en Costa Rica, el uso de feromonas se ha enfocado principalmente en la atracción y captura de diferentes especies de joboto (Phyllophaga spp.) y picudos (Metamasius hemipterus) (Chinchilla et al., 1996; Vargas, 2006). Sin embargo, las feromonas para la atracción de diferentes especies de Spodoptera se encuentran disponibles comercialmente, por lo que su uso para el monitoreo de estos insectos en caña de azúcar es posible y recomendado.

Tanto la caña de azúcar como otros cultivos, se encuentran ante los efectos del cambio climático (aumento en el $\mathrm{CO}_{2}$ atmosférico, cambios en los periodos estacionales e inestables ciclos de tormentas y huracanes), los cuales se ven reflejados en las plagas que afectan los cultivos, por lo que es de esperar que plagas anteriormente consideradas secundarias se conviertan en principales (Trebicki y Finlay, 2019). Consecuentemente, el estudio de la polilla $S$. frugiperda, así como otros miembros del mismo género, se vuelve fundamental. Sin embargo, a la fecha, solo un estudio interno realizado en la Central Azucarera del Tempisque (CATSA), determinó la dinámica de dos especies de Spodoptera observadas en cultivos de arroz anegado alternado con caña de azúcar. El mismo, se enfocó únicamente en los primeros 36 días post-germinación, periodo en el cual se considera que el arroz se encuentra en mayor riesgo de ser afectado por los gusanos cogolleros. Ante este vacío del conocimiento, el presente estudio tuvo como objetivo monitorear las poblaciones de las especies $S$. frugiperda, $S$. sunia y $S$. exigua en una plantación de caña de azúcar.

\section{Materiales y métodos}

\section{Sitio de estudio y condiciones de cultivo}

El estudio se llevó a cabo entre febrero de 2018 y mayo de 2019, en una plantación de caña de azúcar (Saccharum spp.) variedad CC 01-1940 (Viveros, 2018), localizada en Cañas, Guanacaste, Costa Rica (N10²2’31,3”, W085 09'15,3”), a $30 \mathrm{msnm}$. El área utilizada se plantó con arroz anegado durante los dos años previos al inicio del experimento. Quince días antes de la cosecha de arroz se interrumpió el riego, entre ocho y quince días después de la cosecha se realizó la quema de la paja, y ocho días después los residuos de cosecha se incorporaron por medio de una rastra pesada. La siembra con caña de azúcar del área bajo estudio tuvo lugar entre los días 19 y 26 de febrero de 2018, mientras que el experimento se estableció el 06 de febrero del 2018, trece días antes de siembra. Todas las labores agronómicas de preparación y manejo de la plantación correspondieron a las prácticas usuales llevadas a cabo para todas las áreas sembradas en la finca.

\section{Experimento y muestreo}

El experimento correspondió a la colocación de trampas, para la captura de machos de polillas, en bordes de un cañal y su evaluación semanal durante todo el desarrollo del cultivo. Cada trampa se elaboró con un recipiente de plástico con capacidad de 3,78 1, en el que se erigieron dos ventanas laterales, y el fondo se llenó con agua jabonosa, la cual fue sustituida cada semana (Figura 1).

En la tapa de cada recipiente se colgó un dispensador de caucho (ChemTica Internacional, Heredia, Costa Rica) con cada una de las feromonas sexuales formuladas para las siguientes especies: S. frugiperda, S. sunia y $S$. exigua. Cada tipo de feromona correspondió a un tratamiento. Se colocaron diez repeticiones por cada tratamiento. Las feromonas se intercalaron a lo largo del perímetro de la plantación de caña de manera lineal, distanciadas a 15 $\mathrm{m}$ entre cada una. Todas las trampas se colocaron en estacas a aproximadamente 1,5 m sobre el suelo.

Semanalmente se contabilizaron los adultos capturados en cada trampa y se renovó el agua jabonosa en cada recipiente. Las feromonas se reemplazaron cada cuatro semanas en cada uno de los tratamientos. Los datos climatológicos, obtenidos con una estación meteorológica marca Davis y denominada Estación Central, ubicada 


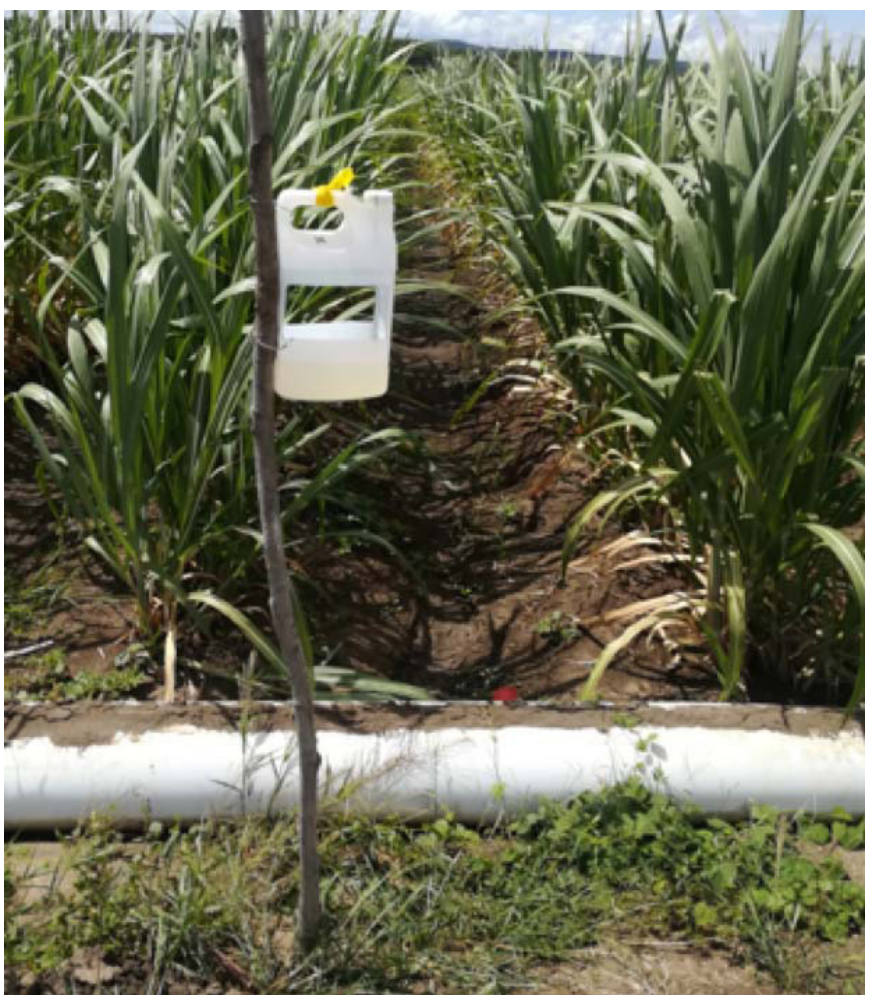

Figura 1. Trampa de agua jabonosa elaborada con un recipiente de un galón de capacidad, colocado en el perímetro del área de monitoreo de poblaciones de Spodoptera spp., en caña de azúcar. Cañas, Costa Rica. 2018-2019.

Figure 1. Soapy water trap made with a one-gallon container, placed in the perimeter of the Spodoptera spp. population monitoring area in sugar cane. Cañas, Costa Rica. 2018-2019.

en La Pradera, Taboga, a una distancia de $4 \mathrm{~km}$ del área del ensayo, se utilizaron para analizar el efecto de la temperatura promedio, la amplitud térmica, la humedad relativa y la precipitación sobre la cantidad de adultos capturados en las trampas monitoreadas. Estas prácticas se realizaron por un período de 58 semanas continuas.

\section{Análisis estadístico}

Todas los datos de capturas se compararon a través del tiempo. Para determinar la especie de Spodoptera spp. predominante en el periodo de estudio se contabilizó el número total de individuos al final del ensayo y se comparó la abundancia de cada especie; para esto se realizó la prueba de normalidad de los datos de KolmogorovSmirnov. Al determinarse que los valores obtenidos no se ajustaron a una distribución normal y que se cumplían los supuestos para la comparación de medias mediante un método no paramétrico, se realizó el análisis de varianza de Kruskal-Wallis y la separación de medias con el método Dwass, Steel, Critchlow-Fligner. El efecto putativo de las variables climatológicas sobre las capturas se evaluó mediante diferentes métodos, en primer lugar se realizaron análisis de correlación de Pearson y regresiones múltiples y finalmente, se realizó un modelo generalizado con efectos mixtos con distribución de Poisson. Los análisis estadísticos se realizaron con SAS Studio (SAS Institute Inc., Cary, NC), Gretl (software estadístico libre, desarrollado por un equipo liderado por Allin Cottrell) y Rstudio (software estadístico libre, RStudio Inc). 


\section{Resultados}

La cantidad total de polillas capturadas durante las 58 semanas de evaluación correspondió a 37816 individuos, de los cuales, aproximadamente el 79,6\% correspondió a capturas de machos de $S$. frugiperda, mientras que las capturas de $S$. sunia y $S$. exigua correspondieron al 19,9 y $0,5 \%$, respectivamente. La cantidad de machos capturados mostró diferencias significativas en la cantidad total de cada una de las especies observadas (Figura 2).

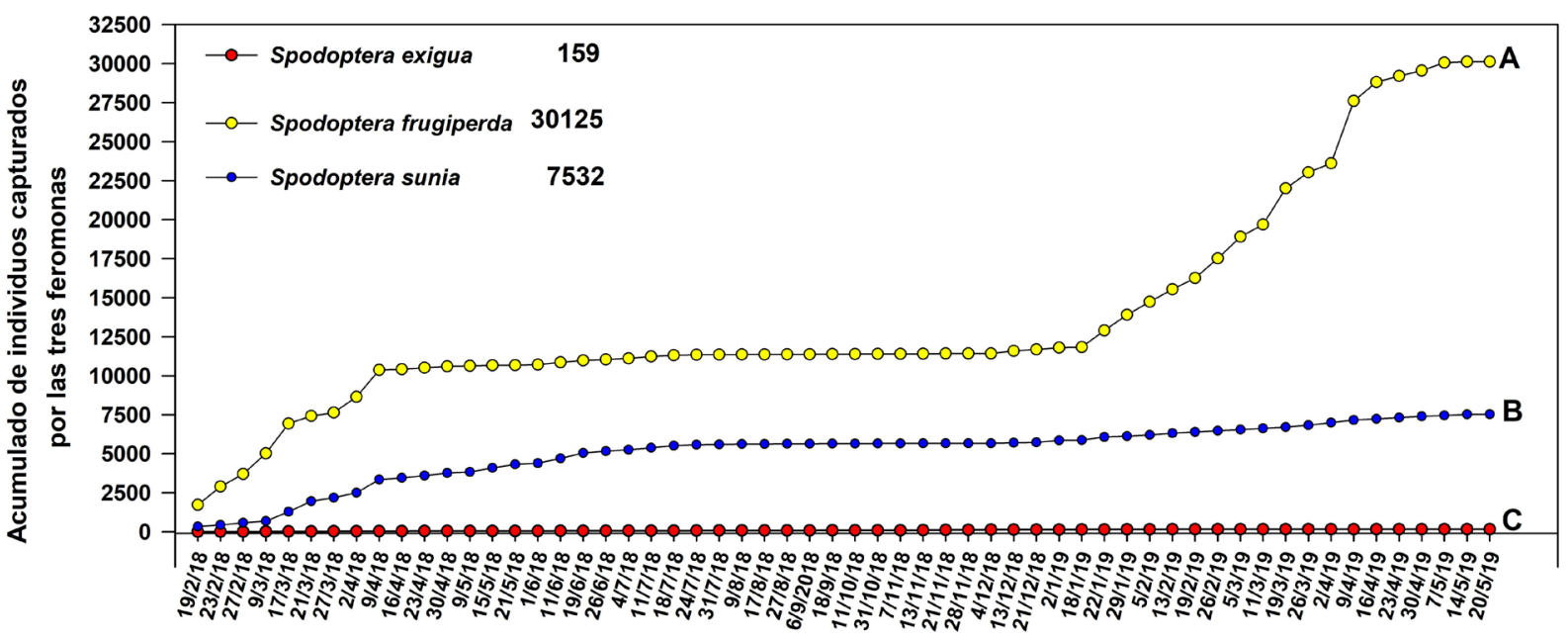

Figura 2. Capturas acumulativas de diferentes especies del género Spodoptera en una plantación de caña de azúcar. Cañas, Costa Rica. 2018-2019.

El número total de polillas capturadas por especie se indica junto a cada leyenda. Letras diferentes al final de cada línea indican diferencias significativas entre los valores totales observados (prueba de Kruskal-Wallis y método Dwass, Steel, Critchlow-Fligner, $\mathrm{p}<0,05$ ).

Figure 2. Cumulative catches of different species of the Spodoptera genus in one sugarcane plantation. Cañas, Costa Rica. $2018-2019$.

The total number of moths caught per species is indicated next to each legend. Different letters at the end of each line indicate significant differences between the overall observed values (Kruskal-Wallis test and Dwass method, Steel, Critchlow-Fligner, $\mathrm{p}<0.05$ ).

Al observar el comportamiento poblacional de las tres especies capturadas durante el 2018 y 2019 , el pico poblacional de $S$. frugiperda estuvo en los primeros cuatro meses del año (enero-abril), mientras que la mayor abundancia de $S$. sunia se observó entre marzo y junio, y finalmente $S$. exigua presentó bajas capturas a lo largo de todo el periodo evaluado (Figura 3). Las variables climatológicas mostraron varias tendencias respecto a las capturas de cada una de las especies estudiadas (Cuadro 1).

La correlación negativa entre capturas de $S$. frugiperda y las variables de humedad relativa y precipitación, indicaron que las poblaciones de esta especie disminuyeron en los periodos de más lluvia, mientras que el caso de $S$. sunia, a pesar que también hubo una correlación negativa con esas variables de clima, no se presentó una disminución tan abrupta en las capturas, debido a que estas fueron bajas. En el caso de S. exigua, todos los factores climatológicos, a excepción de la amplitud térmica, tuvieron una débil correlación negativa, no obstante, las bajas capturas observadas provocaron que estos datos sean difíciles de interpretar (Cuadro 2).

El análisis de regresión múltiple realizado para la abundancia de cada especie, indicó que únicamente para S. exigua los factores climatológicos observados no tuvieron efectos significativos en el número de individuos capturados (ANOVA, $\mathrm{F}=1,10, \mathrm{p}=0,40$ ). 


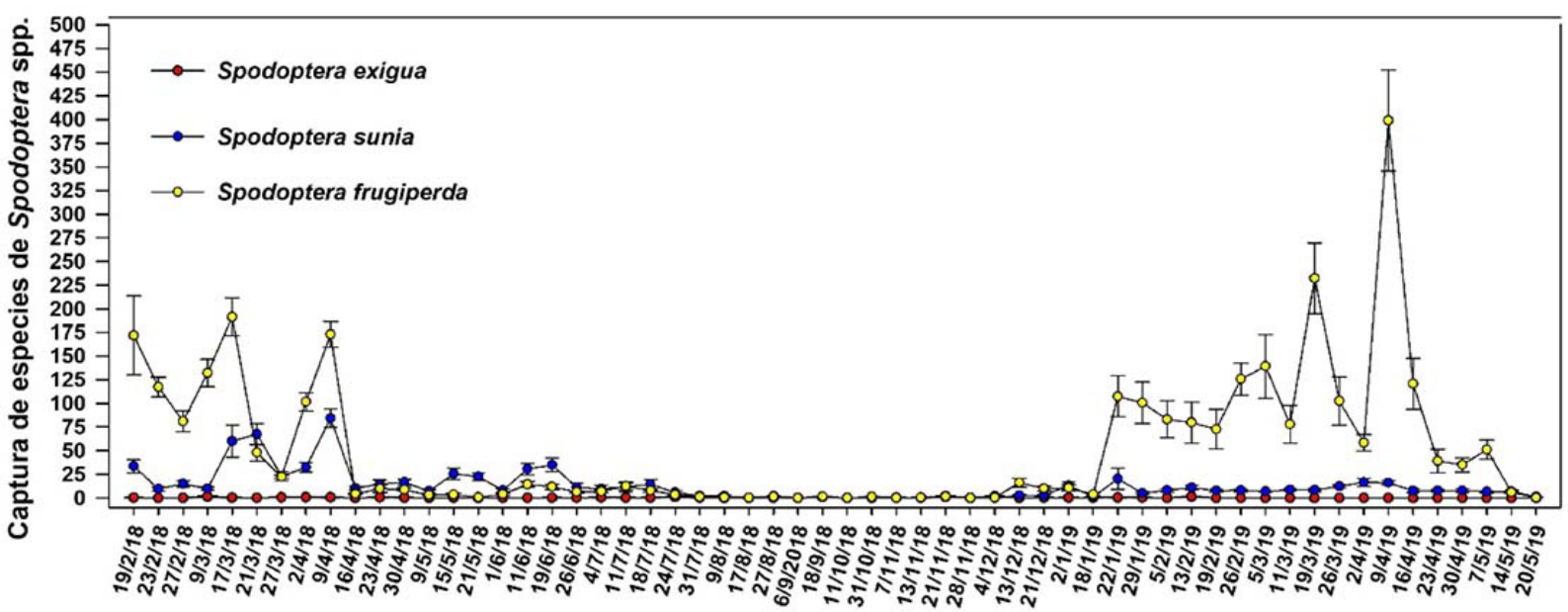

Figura 3. Dinámica poblacional de tres especies del género Spodoptera en una plantación de caña de azúcar. Cañas, Costa Rica.20182019.

Figure 3. Population dynamics of three species of the Spodoptera genus in a sugarcane plantation. Cañas, Costa Rica. 2018-2019.

Cuadro 1. Información climatológica mensual del sitio experimental y captura de diferentes especies de Spodoptera en caña de azúcar durante el periodo 2018-2019. Cañas, Costa Rica.

Table 1. Monthly climatological information of the experimental site and capture of different species of Spodoptera in sugarcane during the period 2018-2019. Cañas, Costa Rica.

\begin{tabular}{|c|c|c|c|c|c|c|c|}
\hline Mes-Año & $\begin{array}{c}\text { Temperatura } \\
\text { promedio }\left({ }^{\circ} \mathbf{C}\right)\end{array}$ & $\begin{array}{c}\text { Humedad } \\
\text { relativa }(\%)\end{array}$ & $\begin{array}{c}\text { Precipitación } \\
\left(\mathbf{m m}^{\mathbf{3}}\right)\end{array}$ & $\begin{array}{c}\text { Amplitud } \\
\text { térmica }\left({ }^{\circ} \mathbf{C}\right)\end{array}$ & $\begin{array}{c}\text { Capturas } S . \\
\text { frugiperda }\end{array}$ & $\begin{array}{c}\text { Capturas } S . \\
\text { sunia }\end{array}$ & $\begin{array}{c}\text { Capturas } S . \\
\text { exigua }\end{array}$ \\
\hline Feb-2018 & 28,9 & 61,3 & 0,1 & 8,4 & 1233,0 & 193,3 & 1,0 \\
\hline Mar-2018 & 29,3 & 62,0 & 0,0 & 8,9 & 1152,0 & 310,0 & 7,0 \\
\hline Abr-2018 & 29,1 & 68,6 & 0,8 & 10,1 & 594,0 & 316,0 & 4,2 \\
\hline May-2018 & 28,8 & 79,6 & 15,6 & 10,3 & 25,0 & 184,0 & 1,0 \\
\hline Jun-2018 & 28,0 & 84,8 & 7,5 & 8,9 & 91,0 & 211,2 & 1,25 \\
\hline Jul-2018 & 27,8 & 79,6 & 3,2 & 8,1 & 63,2 & 87,8 & 4,8 \\
\hline Ago-2018 & 27,9 & 80,5 & 2,9 & 9,2 & 6,0 & 14,0 & 0,7 \\
\hline Sep-2018 & 28,0 & 83,3 & 6,7 & 10,0 & 7,0 & 4,0 & 5,0 \\
\hline Oct-2018 & 26,8 & 87,0 & 14,0 & 8,2 & 6,5 & 4,5 & 0,0 \\
\hline Nov-2018 & 27,4 & 79,2 & 2,1 & 10,1 & 10,0 & 4,0 & 10,0 \\
\hline Dic-2018 & 26,9 & 70,0 & 0,1 & 12,2 & 87,0 & 19,3 & 6,7 \\
\hline Ene-2019 & 26,4 & 65,7 & 0,0 & 13,2 & 555,0 & 99,0 & 3,3 \\
\hline Feb-2019 & 27,2 & 64,2 & 0,0 & 12,7 & 901,2 & 87,0 & 3,3 \\
\hline Mar-2019 & 27,5 & 63,2 & 0,0 & 11,4 & 1303,0 & 111,2 & 0,0 \\
\hline Abr-2019 & 29,6 & 66,5 & 0,0 & 10,9 & 1199,0 & 207,2 & 0,2 \\
\hline Мay-2019 & 28,4 & 83,7 & 5,9 & 8,4 & 190,7 & 48,7 & 0,0 \\
\hline
\end{tabular}


Cuadro 2. Coeficientes de correlación de Pearson entre capturas de diferentes especies de Spodoptera y variables climáticas evaluadas en una plantación de caña de azúcar. Cañas, Costa Rica. 2018-2019.

Table 2. Pearson's correlation coefficients between captures of different Spodoptera species and the weather variables evaluated in a sugarcane plantation located in Cañas, Costa Rica. 2018-2019.

\begin{tabular}{lcccc}
\hline Especie & Temperatura promedio & Humedad Relativa & Precipitación & Amplitud térmica \\
\hline S. frugiperda & 0,4 & $-0,9$ & $-0,6$ & 0,3 \\
S. sunia & 0,7 & $-0,5$ & $-0,2$ & $-0,1$ \\
S. exigua & $-0,1$ & $-0,1$ & $-0,3$ & 0,2 \\
\hline
\end{tabular}

En el caso de S. frugiperda el análisis de regresión múltiple mostró que la temperatura promedio, la amplitud térmica y la humedad relativa fueron factores que afectaron significativamente las capturas de esta especie (ANOVA, $\mathrm{F}=33,41, \mathrm{p}<0,0001$ ), lo cual también se observó en la dinámica de capturas durante el periodo en estudio (Figura 4). Para S. frugiperda el mayor aumento de las poblaciones se dio cuando la amplitud térmica alcanzó el grado de la temperatura más alto (Figura 5), momento en que el ambiente se encontró más caliente, y de este modo

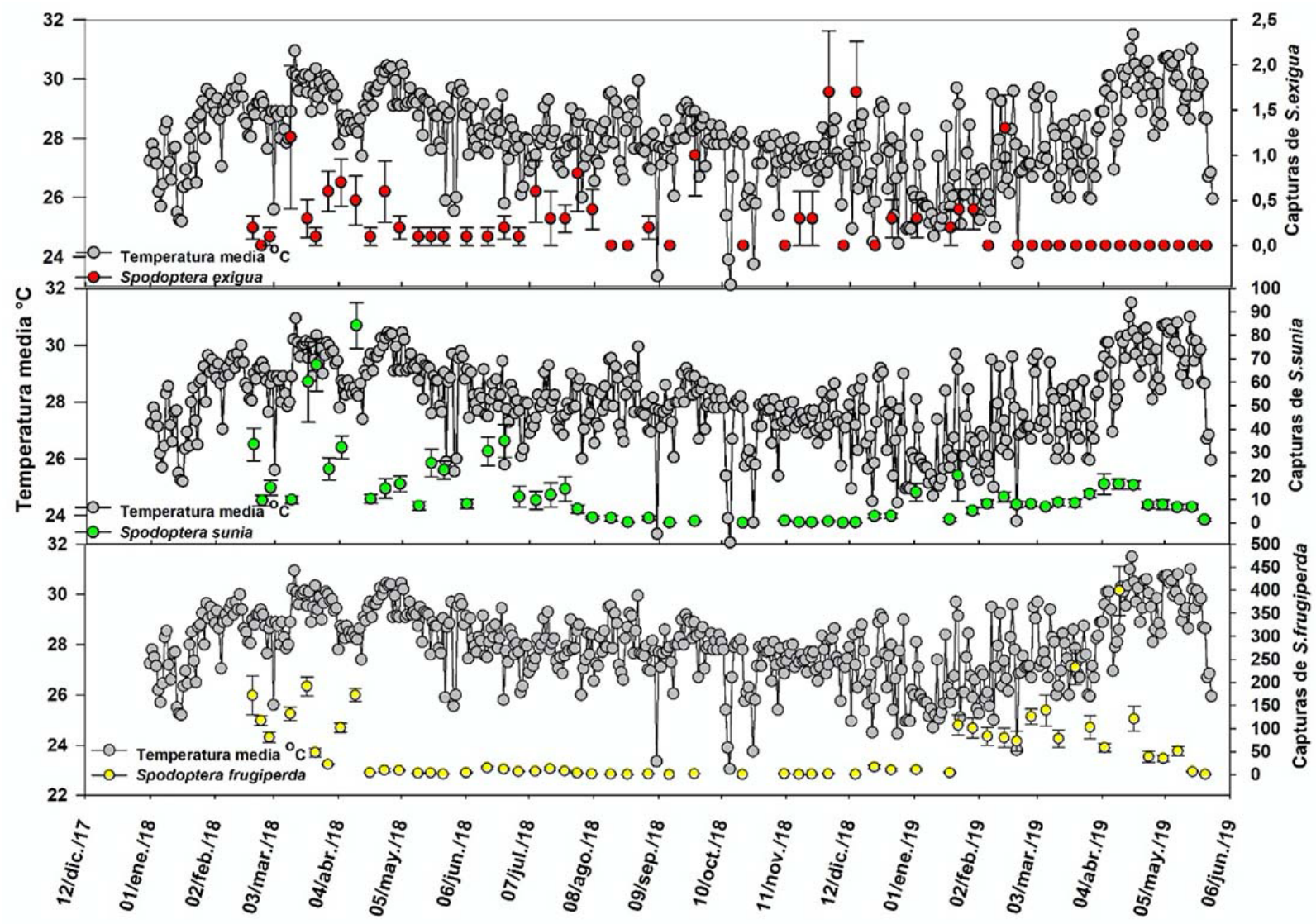

Figura 4. Dinámica poblacional de Spodoptera spp. y su relación con la temperatura promedio en una plantación de caña de azúcar. Cañas, Costa Rica. 2018-2019.

Figure 4. Population dynamics of Spodoptera spp. and its relationship to the average temperature in a sugarcane plantation. Cañas, Costa Rica. 2018-2019. 


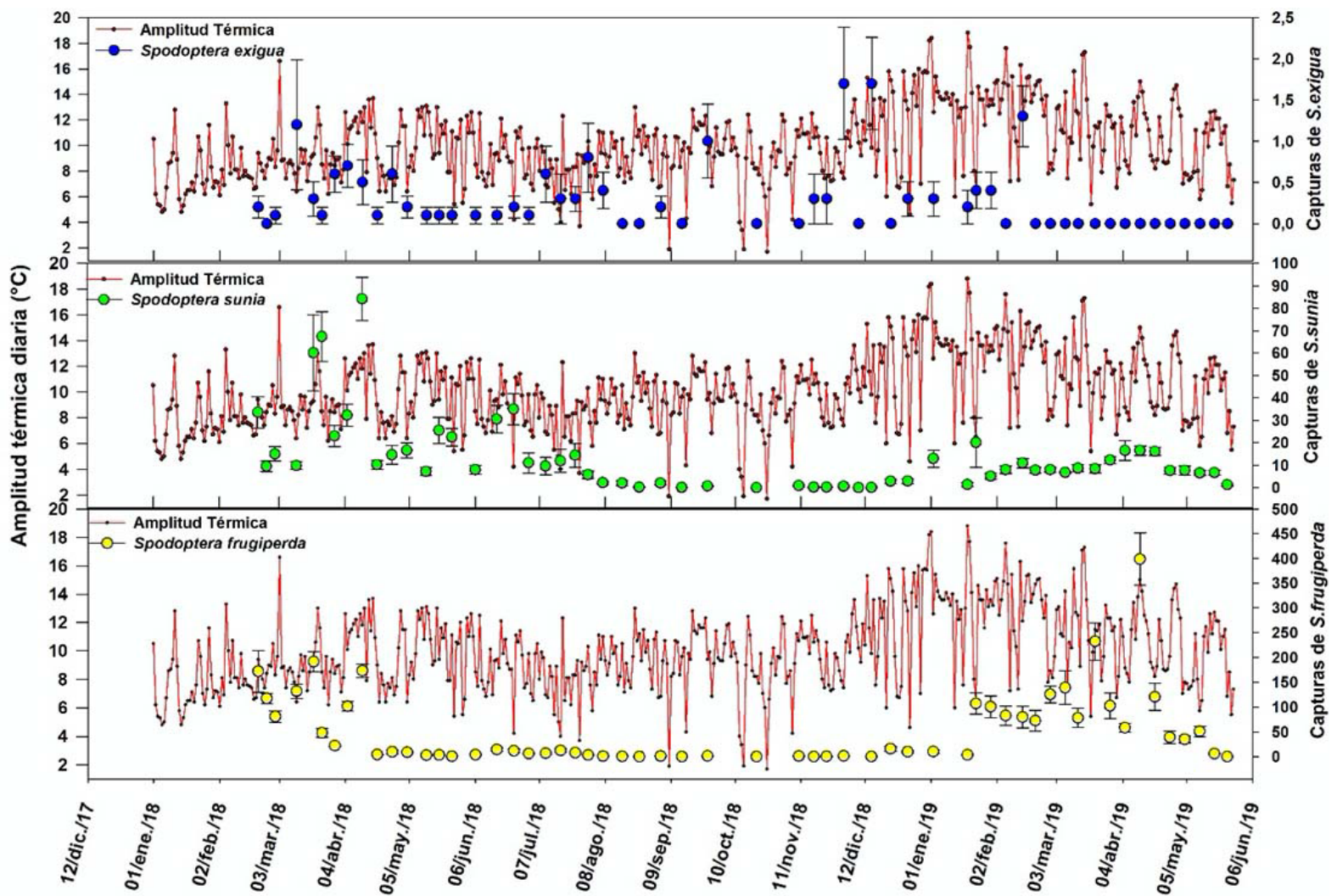

Figura 5. Dinámica poblacional de Spodoptera spp. y su relación con la amplitud térmica en una plantación de caña de azúcar. Cañas, Costa Rica. 2018-2019.

Figure 5. Population dynamics of Spodoptera spp. and its relationship with the thermal amplitude in a sugarcane plantation. Cañas, Costa Rica. 2018-2019.

propició que se acortara el ciclo de vida del insecto plaga (Durán, comunicación personal, 2019). Si se considera que el ciclo de vida de $S$. frugiperda se puede completar en 30 días con temperaturas entre 25 y 30 grados, los resultados indican que se podrían observar en campo aproximadamente unas doce generaciones anuales de este insecto. En el caso de $S$. sunia hubo el mismo comportamiento pero menos marcado, pero es probable observar al menos doce generaciones anuales, porque su ciclo dura 30 días a una temperatura óptima de $26{ }^{\circ} \mathrm{C}$. Para $S$. exigua, al existir capturas en niveles muy bajos, realmente no es posible correlacionar la temperatura con las poblaciones de machos observados.

Respecto a la humedad, se observó que al disminuir el porcentaje de humedad relativa la población de adultos aumentó en las especies estudiadas (Figura 6). Aunque el análisis de regresión lineal no señaló la precipitación como un factor importante, el análisis de correlación indicó, que al igual que la humedad relativa, tuvo un fuerte efecto negativo en la captura de machos de $S$. frugiperda. Se denotó (Figura 7) cómo el menor número de capturas coincidió con los meses más lluviosos y de menor variación térmica para las tres especies en estudio, por lo que el impacto de las lluvias sobre las condiciones agroecológicas y sobre la reproducción y migración de Spodoptera 


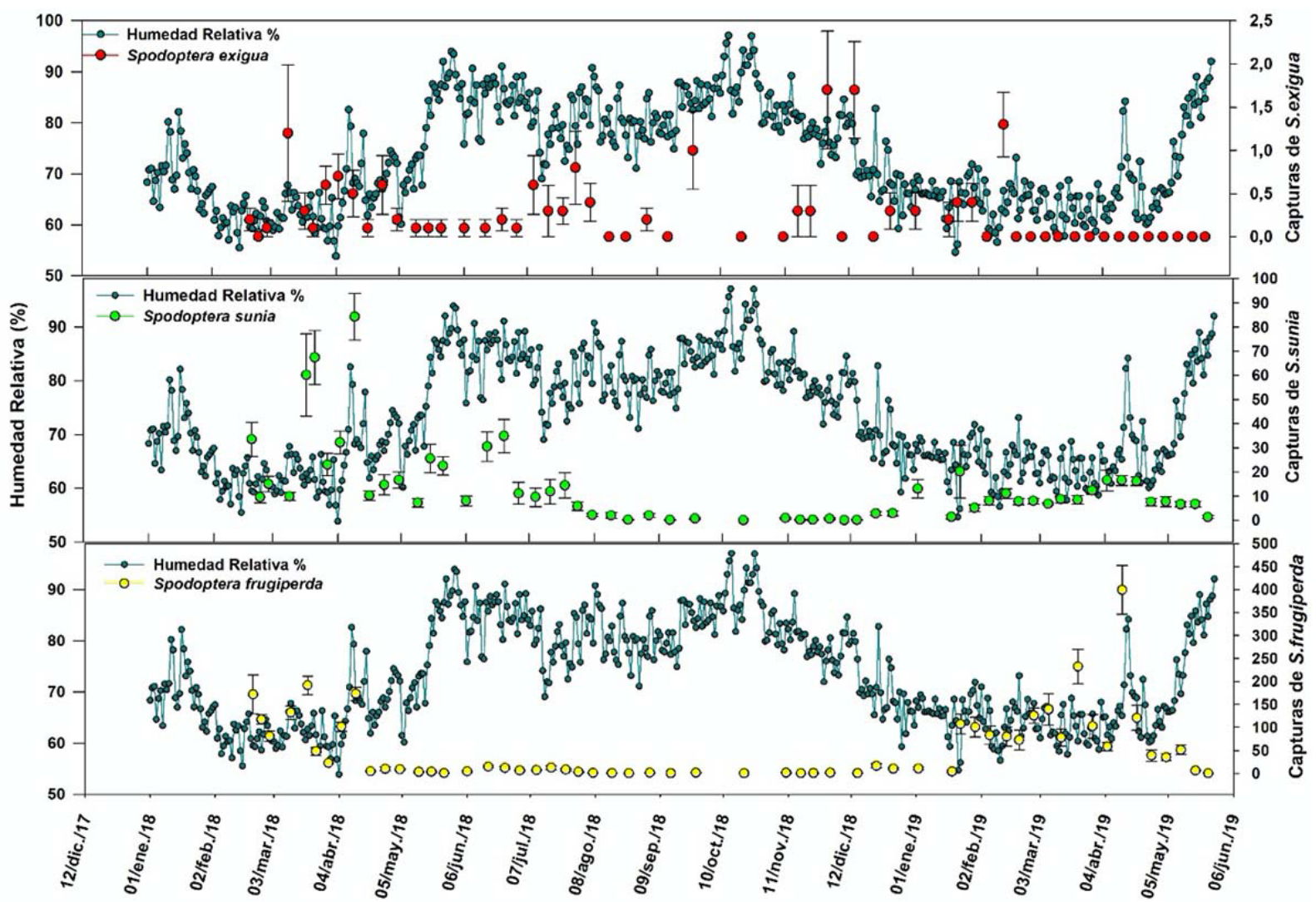

Figura 6. Dinámica poblacional de Spodoptera spp. y su relación con el porcentaje de humedad relativa del ambiente en una plantación de caña de azúcar. Cañas, Costa Rica. 2018-2019.

Figure 6. Population dynamics of Spodoptera spp. and its relationship with the percentage of an environment relative humidity in a sugarcane plantation. Cañas, Guanacaste. 2018-2019.

spp., fue predominante. En el caso de $S$. sunia, los resultados del análisis de regresión múltiple indicaron que únicamente la humedad relativa tuvo efectos significativos en la captura de machos de esta especie (Prueba $\mathrm{T}, \mathrm{t}=-$ $2,46, \mathrm{p}=0,03)$.

El análisis mediante la prueba del modelo lineal (lm) demostró que, tanto la amplitud térmica (AT) como la temperatura promedio (TM) y sus interacciones fueron significativas en el efecto sobre la captura de machos de Spodoptera spp. Por tanto, se procedió a realizar un análisis por medio del modelo generalizado mixto (glm), con distribución de Poisson para las variables de amplitud térmica (AT) y temperatura media (TM) para las capturas de las tres especies. Para S. frugiperda y $S$. sunia hubo significancia en todos sus coeficientes, esto describe como estas dos variables climáticas influyeron positivamente en la dinámica o en la presencia de adultos en vuelo, por su parte en S. exigua el total de capturas fue muy bajo, por lo tanto, no correlacionó estadísticamente ni presentó significancia para ninguna variable climática (Cuadro 3). 


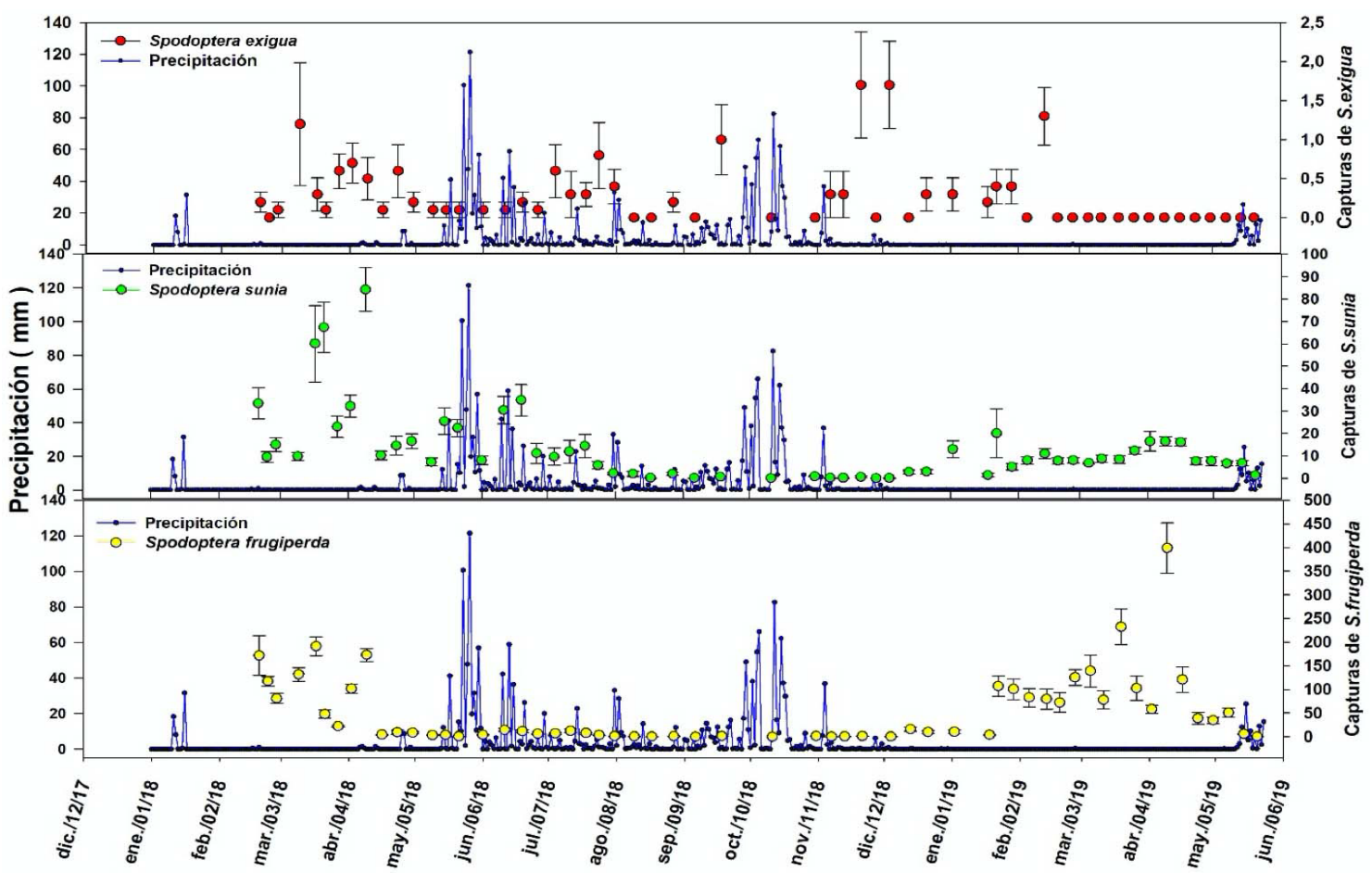

Figura 7. Dinámica poblacional de Spodoptera spp. relacionada con la precipitación en una plantación de caña de azúcar. Cañas, Costa Rica. 2018-2019.

Figure 7. Population dynamics of Spodoptera spp. related to precipitation in a sugarcane plantation. Cañas, Costa Rica. $2018-2019$.

Cuadro 3. Análisis con modelos generalizados mixtos con distribución de Poisson entre la amplitud térmica (AT), temperatura media (TM) y su correlación con las capturas de Spodoptera spp. evaluadas en una plantación de caña de azúcar localizada en Cañas, Costa Rica. 2018-2019.

Table 3. Analysis with mixed generalized models with Poisson distribution between thermal amplitude (AT), average temperature (TM), and its correlation with the captures of Spodoptera spp. evaluated in a sugarcane plantation located in Cañas, Costa Rica. 2018-2019.

\begin{tabular}{|c|c|c|c|c|c|}
\hline Especies & S. frugiperda & $\operatorname{Pr}(>|z|)$ & S. sunia & $\operatorname{Pr}(>|z|)$ & S. exigua \\
\hline \multirow[t]{2}{*}{ Constante } & $-151,70$ & $* * *$ & 87,68 & $* * *$ & -167 \\
\hline & $2,00 \mathrm{E}-16$ & & $1,24 \mathrm{E}-07$ & & 0,2970 \\
\hline \multirow[t]{2}{*}{ AT } & 17 & $* * *$ & $-9,547$ & $* * *$ & $-6,99$ \\
\hline & $2,00 \mathrm{E}-16$ & & $8,22 \mathrm{E}-10$ & & 0,6260 \\
\hline \multirow[t]{2}{*}{$\mathrm{TM}$} & 7,79 & $* * *$ & $-4,514$ & $* * *$ & 12,87 \\
\hline & $2,00 \mathrm{E}-16$ & & $3,23 \mathrm{E}-05$ & & 0,2400 \\
\hline \multirow[t]{2}{*}{ AT_2 } & $-0,3748$ & $* * *$ & 0,1997 & $* * *$ & 0,2024 \\
\hline & $2,00 \mathrm{E}-16$ & & $1,02 \mathrm{E}-07$ & & 0,5710 \\
\hline \multirow[t]{2}{*}{ TM_2 } & $-0,0793$ & $* * *$ & 0,0507 & $* *$ & $-0,2488$ \\
\hline & $4,31 \mathrm{E}-14$ & & $5,64 \mathrm{E}-03$ & & 0,2020 \\
\hline \multirow[t]{2}{*}{$\mathrm{AT} * \mathrm{TM}$} & $-0,6155$ & $* * *$ & 0,351 & $* * *$ & 0,265 \\
\hline & $2,00 \mathrm{E}-16$ & & $1,22 \mathrm{E}-09$ & & 0,6180 \\
\hline \multirow[t]{2}{*}{$\mathrm{AT}^{*} \mathrm{TM}^{\wedge} 2$} & 0,0005 & $* * *$ & $-0,0003$ & $* * *$ & $-0,0003$ \\
\hline & $2,00 \mathrm{E}-16$ & & $1,35 \mathrm{E}-07$ & & 0,5490 \\
\hline
\end{tabular}

Nota: error estándar entre paréntesis. *** Altamente significativo al $1 \%$, ** significativo al $5 \%$ / Note: standard error in parentheses. *** Highly significant at $1 \%, * *$ Significant at $5 \%$. 


\section{Discusión}

El desarrollo de malezas cerca de la plantación de caña se ha señalado como el principal factor que favorece el desarrollo del cogollero del maíz. Esto generalmente se debe a que las hembras grávidas colocan sus huevos en malezas cercanas a la plantación cuando la caña es aún pequeña (tres meses postemergencia), pero que al darse las aplicaciones tardías de herbicidas presionan a las poblacionas a migrar y utilizar la caña de azúcar como hospedero, en donde en casos extremos las larvas puede alimentarse del $100 \%$ de la lámina foliar o incluso barrenar los tallos y nuevos brotes (Gómez y Lastra, 1995).

La especie $S$. sunia, no es una plaga tradicionalmente asociada a caña de azúcar y su mayor abundancia se da en los cultivos de melón (Cucumis melo) y sandía (Citrullus lanatus), donde puede constituir una plaga de importancia económica (Alpízar-Monge y Rodríguez-Valverde, 1997). En el caso de S. exigua, su presencia en caña de azúcar es probablemente meramente facultativa, pues es un insecto polífago, capaz de alimentarse de más de noventa especies de plantas, pero que sin embargo, presenta una gradiente preferencial, teniendo el algodón y la remolacha como hospederos preferidos (Greenberg et al., 2001).

Las feromonas sexuales de polillas son capaces de atraer machos en rangos que varían desde decenas hasta unas centenas de metros (Allison y Cardé, 2016). Si se considera que trampas de marcaje y recaptura de $S$. frugiperda han mostrado que las trampas de feromona son capaces de atraer adultos hasta un máximo de $806 \mathrm{~m}$ (Vilarinho et al., 2011), y que el área utilizada para el estudio no tiene plantaciones de maíz, melón, y algodón cercanos, es poco probable que los individuos capturados de las tres especies en estudio tuvieran su origen en áreas y hospederos desligados de la producción cañera. Es posible que las diferentes especies de Spodoptera observadas correspondieron a poblaciones desarrolladas en parte o en su totalidad en la caña de azúcar o en las arvenses presentes naturalmente en estas plantaciones. Un meta-análisis reciente ha demostrado que el número de especies de plantas hospederas de $S$. frugiperda es mucho mayor a lo que se consideraba anteriormente. Se ha determinado que en las Américas existen 356 especies hospederas del cogollero del maíz (Montezano et al., 2018), de las cuales algunas como Cenchrus echinatus, Digitaria sanguinalis, Echinochloa colona, Sorghum halepense, Cynodon dactylon, Ipomoea triloba y otras, son malezas importantes en el cultivo de la caña de azúcar (Espinoza et al., 2013). Es probable, que muchas de estas malezas sean también hospederos comunes aún no determinados de las tres especies de Spodoptera observadas en el presente estudio.

El desarrollo del cogollero del maíz es óptimo a una temperatura promedio de $28{ }^{\circ} \mathrm{C}$, pero se ha observado que se acorta el ciclo conforme la temperatura se acerca a los $30{ }^{\circ} \mathrm{C}$ (Simmons, 1993; CABI, 2017; Early et al., 2018). Como se observa en la Figura 4, durante la mayoría del tiempo las temperaturas promedio se mantuvieron en condiciones que favorecieron el desarrollo de la plaga, pero aun así las temperaturas más bajas tendieron a asociarse con menores capturas. Es probable que esta dinámica esté relacionada directamente con la amplitud térmica, la cual por definición es la diferencia entre la temperatura máxima y la mínima de un mismo día, y que afecta directamente el comportamiento de los insectos, debido a que tiene efectos directos sobre los procesos bioquímicos que cada especie realiza. Este factor relaciona directamente la temperatura que pudo quedar atrapada por efectos de la capa límite de la atmósfera y de factores como la nubosidad y $\mathrm{CO}_{2}$ (efecto invernadero), por ende influye directamente en la humedad relativa y en el comportamiento de los seres vivos. Este factor es importante pues indica que si bien la temperatura promedio fue favorable para el insecto, el detalle de los cambios graduales térmicos observados durante el día también tuvo un efecto en el desarrollo de la plaga.

El propio comportamiento reproductivo de $S$. frugiperda es variable dependiendo de la temperatura en el momento de cortejo (Simmons y Marti, 1992). Además, experimentos realizados en laboratorio han demostrado que el gusano cogollero es capaz de tener ciclos más cortos y con más altas tasas de supervivencia cuando existen temperaturas variables en comparación a temperaturas constantes (Barfield y Ashley, 1987). El efecto negativo de la humedad relativa (promedio de $74 \%$ ) en el desarrollo de este insecto resulta interesante, pues existen reportes 
en los cuales se indica que la supervivencia, el tiempo de desarrollo y la aparición de deformidades, son factores no afectados por humedades relativas fluctuantes entre 20 y $100 \%$ (Simmons, 1993).

La temperatura, la precipitación y el tipo de suelo como factores que pueden afectar el desarrollo de Spodoptera fueron determinados por Sims (2008). Debido a que estas polillas pupan en el suelo después de completar sus instares larvales, las condiciones de alta humedad y precipitación conllevan efectos negativos como hipoxia o desarrollo de hongos, mohos u otros que afectan negativamente la emergencia de adultos. Además, el exceso de humedad y la alta precipitación pueden saturar los espacios foliares en los que se alimentan las larvas, lo que causa la muerte por ahogamiento o la caída fuera de las plantas (Van-Huis, 1981).

Existen reportes de que el ciclo biológico de $S$. sunia se acorta conforme se incrementa la temperatura, con un desarrollo óptimo cerca de los $26{ }^{\circ} \mathrm{C}$ (La-Rosa et al., 1992), no obstante, este efecto no se observó en este experimento. Aunque, no se encontraron reportes sobre el efecto de la humedad relativa sobre el desarrollo de esta especie, es de esperar que al igual que en $S$. frugiperda, condiciones de baja humedad relativa favorezcan la emergencia de mayor cantidad de adultos de las pupas que completan su ciclo en el suelo, y poblaciones más altas de estas polillas durante los meses más secos.

Estudios realizados en Chiapas, México, mostraron dos picos poblacionales bien definidos para la captura de S. frugiperda, el primero en la época seca (entre enero y marzo) y el segundo en la época lluviosa (entre junio y septiembre), pero con variabilidad entre los diferentes años en estudio (Rojas et al., 2004). En un estudio similar realizado en Irapuato, México, se demostró la prevalencia de dos picos poblacionales, definidos entre julio y agosto y, posteriormente entre octubre y noviembre (Salas-Araiza et al., 2018). En el caso de países templados, dado que esta plaga no es capaz de sobrevivir periodos extensos de congelación, se dan picos migratorios hacia regiones de climas más cálidos durante momentos específicos del año (Westbrook et al., 2016). En los resultados observados en el presente estudio se consideró un solo pico poblacional para $S$. frugiperda que abarcó toda la época seca de la región estudiada. Estos resultados demuestran que si bien las estimaciones de la dinámica poblacional de las especies en estudio proveen una herramienta para conocer mejor el desarrollo de la plaga, también tienden a ser dependientes de la región y el año en estudio, y esto no se debe únicamente a las variables climáticas si no también a factores como la presencia de hospederos alternos y/o enemigos naturales. Tanto la temperatura como la precipitación son factores que afectan la abundancia de larvas de Spodoptera y de sus parasitoides en la región Noroeste de Argentina (Murúa et al., 2006).

Las tres especies estudiadas, no son consideradas plagas de importancia para el cultivo de la caña de azúcar; no obstante, y como lo muestran los resultados obtenidos en este estudio, la fluctuación de las condiciones climáticas y la presencia de malezas que sirven como hospederos alternos, son dos factores que pueden propiciar el establecimiento y desarrollo de poblaciones de estos insectos fitófagos que pudiesen poner en peligro las plantaciones más jóvenes de caña de azúcar. Los resultados de los diferentes análisis realizados indican que la temperatura promedio y particularmente, la amplitud térmica son factores que una y otra vez se correlacionaron con las capturas de $S$. frugiperda. En periodos de poca lluvia se observó mayor cantidad de machos de $S$. fugiperda y $S$. sunia, por lo que es posible predecir que las sequías y el desfase anual de precipitaciones pueda incrementar los ataques en plantaciones de caña de azúcar (Early et al., 2018). El cambio climático se ha demostrado que se correlaciona con la aparición de brotes de patógenos y plagas fuera de las temporadas anuales esperadas, la migración de especies invasoras, la reaparición de problemas fitosanitarios previamente controlados y la transición de plagas en orden de importancia (Trebicki y Finlay, 2019); por tanto, se hace necesario el monitoreo constante de las dinámicas poblacionales de estas polillas mediante el uso de trampas con feromonas, pues permitirá predecir y determinar los riesgos asociados al cultivo de ambos, caña de azúcar y arroz anegado. 


\section{Conclusiones}

Las capturas de machos de las diferentes especies de Spodoptera, mostraron que $S$. frugiperda fue la especie más abundante durante los dos periodos secos analizados (febrero-mayo), pero existieron periodos durante el desarrollo de la caña de azúcar en los cuales $S$. sunia fue más abundante. Estas diferencias estuvieron relacionadas con las variables climatológicas y su efecto en cada especie, periodos secos influyeron en una mayor captura de $S$. frugiperda, temperaturas más altas se correlacionaron con más altas poblaciones de $S$. sunia, mientras que la alta precipitación y la alta humedad relativa se correlacionaron con bajas poblaciones de todas las especies en estudio.

\section{Agradecimientos}

Los autores agradecen a los señores Johnny Ruiz y José Molina del Ingenio Taboga S.A., por su colaboración en el desarrollo de este proyecto.

\section{Literatura citada}

Allison, J.D., and R.T. Cardé. 2016. Pheromone communication in moths: evolution, behavior, and application. University of California Press, CA, USA.

Alpízar-Monge, D., y C.L. Rodríguez-Valverde. 1997. El uso de feromonas y otros atrayentes de insectos de los cultivos de Costa Rica. En: IICA, y CATIE, editores, IV Congreso Costarricense de Entomología. IICA, y CATIE, San José, CRI. p11-17.

Barfield, C.S., and T.R. Ashley. 1987. Effects of corn phenology and temperature on the life cycle of the fall armyworm, Spodoptera frugiperda (Lepidoptera: Noctuidae). FL. Entomol. 70:110-116. doi:10.2307/3495097

Baudron, F., M.A. Zaman-Allah, I. Chaipa, N.Chari, and P. Chinwada. 2019. Understanding the factors influencing fall armyworm (Spodoptera frugiperda JE Smith) damage in African smallholder maize fields and quantifying its impact on yield. Crop Prot. 120:141-150. doi:10.1016/j.cropro.2019.01.028

CABI. 2017. Scientists discover new crop destroying armyworm is now 'spreading rapidly' in Africa. CABI, Wallingford, GBR.

Chinchilla, C.M., A.C. Oehlschlager, and J.M. Bulgarelli. 1996. A pheromone based trapping system for Rhynchophorus palmarum and Metamasius hemipterus. ASD Oil Palm Papers 12:11-17.

Chormule, A., N. Shejawal, C. M.Sharanabasappa, R. Asokan, and H.M. Swamy. 2019. First report of the fall armyworm, Spodoptera frugiperda (JE Smith) (Lepidoptera, Noctuidae) on sugarcane and other crops from Maharashtra, India. J. Entomol. Zool. Stud. 7:114-117.

Early, R., P. Gonzalez-Moreno, S.T. Murphy, and R. Day. 2018. Forecasting the global extent of invasion of the cereal pest Spodoptera frugiperda, the fall armyworm. NeoBiota 40:28165. doi:10.3897/neobiota.40.28165

Espinoza, G., C. Hernández, y J. Morales. 2013. Manual de malezas y catálogo de -herbicidas para el cultivo de la caña de azúcar en Guatemala. Centro Guatemalteco de Investigación y Capacitación de la Caña de Azúcar, Escuintla, GTM.

FAO, and CABI. 2019. Community-based fall armyworm (Spodoptera frugiperda) monitoring, early warning and management: Training of trainers manual. FAO, and CABI, USA. 
Gómez, L.A., y L.A. Lastra. 1995. Insectos asociados con la caña de azúcar en Colombia. En: C. Cassalett-Dávila et al., editores. El cultivo de la caña en la zona azucarera de Colombia. Centro de investigación de la caña de azúcar en Colombia (CENICAÑA), Cali, COL. p. 237-258.

Greenberg, S.M., T.W. Sappington, B.C Legaspi, T.X Liu, and M. Setamou. 2001. Feeding and life history of Spodoptera exigua (Lepidoptera: Noctuidae) on different host plants. Ann. Entomol. Soc. Am. 94:566-575.

LAICA (Liga Agrícola Industrial de la Caña de Azúcar). 2019. Informe de resultados 2018. Programa de Fitosanidad - Manejo de Plagas. LAICA, San José, CRI.

La-Rosa, J., R. Cabrera, y R. Vera. 1992. Influencia de la temperatura en el desarrollo de Spodoptera sunia (Lepidoptera, Noctuidae) en tomate" Campbell-28". Protección de Plantas 2(3):13-21.

Montezano, D.G., A. Specht, D.R. Sosa-Gómez, V.F. Roque-Specht, ,J.C. Sousa-Silva, S.V.D. Paula-Moraes, J.A. Peterson, and T.E. Hunt. 2018. Host plants of Spodoptera frugiperda (Lepidoptera: Noctuidae) in the Americas. Afr. Entomol. 26:286300. doi:10.4001/003.026.0286

Murúa, G., J. Molina-Ochoa, and C. Coviella. 2006. Population dynamics of the fall armyworm, Spodoptera frugiperda (Lepidoptera: Noctuidae) and its parasitoids in northwestern Argentina. FL. Entomol. 89:175-183. doi:10.1653/00154040(2006)89[175:PDOTFA]2.0.CO;2

Rojas, J.C., A. Virgen, and E.A. Malo. 2004. Seasonal and nocturnal flight activity of Spodoptera frugiperda males (Lepidoptera: Noctuidae) monitored by pheromone traps in the coast of Chiapas, Mexico. FL. Entomol. 87:496-503. doi:10.1653/00154040(2004)087[0496:SANFAO]2.0.CO;2

Salas-Araiza, M.D., O.A., Martínez-Jaime, R. Guzmán-Mendoza, M.A. González-Márquez, and Á. Ávila-López, Á. 2018. Fluctuación poblacional de Spodoptera frugiperda (JE Smith) y Spodoptera exigua (Hubner) (Lepidoptera: Noctuidae) mediante el uso de feromonas en Iraputo, Gto. Mex. Entomol. Mex. 5:368-374.

Salazar, J.D., J.F. González, E. Cadet, R. Oviedo, y C.E. Sáenz. 2017. Catálogo de identificación de plagas del cultivo de la caña de azúcar en Costa Rica. LAICA, Grecia, CRI.

Sims, S.R. 2008. Influence of soil type and rainfall on pupal survival and adult emergence of the fall armyworm (Lepidoptera: Noctuidae) in Southern Florida. J. Entomol. Sci. 43: 373-380. doi:10.18474/0749-8004-43.4.373

Simmons, A.M. 1993. Effects of constant and fluctuating temperatures and humidities on the survival of Spodoptera frugiperda pupae (Lepidoptera: Noctuidae). FL. Entomol. 76:333-340. doi:10.2307/3495733

Simmons, A.M., and O.G. Marti. 1992. Mating by the fall armyworm (Lepidoptera: Noctuidae): Frequency, duration, and effect of temperature. Environ. Entomol. 21: 371-375. doi:10.1093/ee/21.2.371

Sparks, A.N. 1979. A review of the biology of the fall armyworm. FL. Entomol. 62:82-87. doi:10.2307/3494083

Srikanth, J., N. Geetha, B. Singaravelu, T. Ramasubramanian, P. Mahesh, L. Saravanan, K.P. Salin, N. Chitra, and M. Muthukumar. 2018. First report of occurrence of fall armyworm Spodoptera frugiperda in sugarcane from Tamil Nadu, India. J. Sugarcane Res. 8(2):195-202.

Trebicki, P., and K. Finlay. 2019. Pests and diseases under climate change; its threat to food security. In: S.S. Yadav, editors, Food security and climate change. Wiley Blackwell, Chinnai, IND. p. 229-249. doi:10.1002/9781119180661.ch11

Vargas, A.J. 2006. Evaluación de trampa tipo balde para el uso de feromona sexual en captura de adultos de Phyllophaga elenans (Coleoptera: Scarabaeidae), en el cultivo de la caña de azúcar. En: S.M. Chaves, editor, XVI Congreso de la Asociación de Técnicos Azucareros de Centro América (ATACA) y XVI Congreso de la Asociación de Técnicos Azucareros de Costa Rica (ATACORI). Tomo 1, vol. 2. ATACORI, San José, CRI. p. 487-491. 
Van-Huis, A. 1981. Integrated pest management in the small farmer's maize crop in Nicaragua. Meded. Dr. Diss., Landbouwhogeschool Wageningen, Wageningen, NLD.

Viveros, V.C. 2018. Características agronómicas y de productividad de la variedad Cenicaña Colombia (CC) 01-1940. Serie Técnica $\mathrm{N}^{\circ} 40$. Cenicaña, Cali, COL.

Vilarinho, E.C., O.A., Fernandes, T.E., Hunt, and D.F. Caixeta. 2011. Movement of Spodoptera frugiperda adults (Lepidoptera: Noctuidae) in maize in Brazil. FL Entomol. 94:480-488. doi:10.2307/41336431

Westbrook, J.K., R.N., Nagoshi, R.L., Meagher, S.J., Fleischer, and S. Jairam. 2016. Modeling seasonal migration of fall armyworm moths. Int. J. Biometeorol. 60:255-267. doi:10.1007/s00484-015-1022-x 